\title{
Aligning the ICD-11 classification of disorders due to substance use with global service needs
}

\author{
V. Poznyak $^{1 *}$, G. M. Reed ${ }^{1,2,3}$ and M. E. Medina-Mora ${ }^{2}$ \\ ${ }^{1}$ Department of Mental Health and Substance Abuse (MSD), World Health Organization, Geneva, Switzerland \\ 2 National Institute of PsychiatryRamón de la Fuente Muñiz, Mexico City, Mexico \\ ${ }^{3}$ Global Mental Health Program, Columbia University Medical Center, New York, NY, USA
}

\begin{abstract}
The use of psychoactive, potentially dependence-producing substances is highly prevalent around the world, and contributes substantially to global disease burden. There is a major gap between the need for treatment for substance use disorders. Changes proposed for the classification of substance use disorders in the Eleventh Revision of the International Classification of Diseases and Related Health Problems, based on a public health approach, have important implications for the conceptualisation, structure and availability of services. These include: (1) an updated and expanded range of substance classes; (2) greater specification of different harmful patterns of substance use, which may be continuous or episodic and recurrent; (3) a new category to denote single episodes of harmful use; (4) a category describing hazardous use of substances; and (5) simplification of diagnostic guidelines for substance dependence. This paper describes these changes and the opportunities they present for improved prevention, treatment, monitoring and health policy.
\end{abstract}

First published online 4 December 2017

Key words: Classification, ICD-11, substance abuse, substance use disorders, treatment.

The use of psychoactive, potentially dependenceproducing substances is highly prevalent around the world. About 2.6 billion people around the world approximately $52 \%$ of the world's population over 15 years of age - have consumed alcohol in their lives, and about 1.9 billion people during the past 12 months (World Health Organization, 2014a, b). More than 1 billion people in the world, or $21 \%$ of the world's population over the age of 15 , are estimated to be tobacco smokers (World Health Organization, 2015). Approximately 250 million people, or $5 \%$ of the world's adult population, were estimated to have used other psychoactive drugs at least once in 2015 (United Nations Office on Drugs and Crime, 2017).

Moreover, the health consequences of substance use and substance use disorders are a major global problem. The results of a comprehensive assessment of risk factors for poor health (GBD 2015 Risk Factors Collaborators, 2016) indicate that tobacco and alcohol use rank among the top ten health risk factors in terms of disease burden (2nd and 9th places). Other drugs use ranks 24 th of the 79 risk factors assessed in its contribution to global disease burden. Data from this study also indicate that psychoactive substance use has played an increasing role in morbidity and mortality since 1990.

* Address for correspondence: Dr Vladimir Poznyak, Department of Mental Health and Substance Abuse (MSD), World Health Organization, 20 Avenue Appia, CH-1211 Geneva, Switzerland.

(Email: pozynakv@who.int)
The public health impact and disease burden attributable to psychoactive substance use are obviously not limited to substance use disorders; significant harm is associated with substance intoxication and healthdamaging effects of substances or their methods of administration. Examples of such harm include traffic injuries, lung cancer related to smoking, liver cirrhosis related to alcohol and blood-borne infections related to injecting drug use (World Health Organization, 2004).

Development of a diagnosable substance use disorder generally aggravates all health risks associated with substance use. In spite of the fact that there are a variety of effective and cost-effective interventions for substance use disorders, there is a major gap between the need for treatment and the delivery of services and overall rates of treatment are low. According to the results of the World Mental Health Survey, based on data from 15 countries of various income levels, the proportion of individuals with lifetime substance use disorders receiving treatment within 1 year of the onset of the disorder ranged from $0.9 \%$ in Mexico to $18.6 \%$ in Spain (Wang, Aguilar-Gaxiola et al. 2007). There are also major treatment delays, during which the psychosocial consequences, costs and disease burden for individuals with substance use disorders, the people around them, and for society accumulate, including disturbances in family relationships, reduced educational attainment and occupational performance, entanglement with the criminal justice system, in addition to dramatic 
increases in medical costs (McLelland et al., 2000; Chisholm \& Saxena, 2012; Strang et al., 2012). The World Mental Health Survey found that delay in treatment after the onset of a substance use disorder ranged from 6 years in Spain to 18 years in Belgium (Wang, Angermeyer et al. 2007).

The available data indicate that efforts to reduce the disease burden attributable to psychoactive substance use should include prevention and population-based public health strategies as well as effective treatment for substance use disorders. What is needed is a broad spectrum of interventions designed for implementation by different sectors of health professionals, and, where appropriate, non-professionals, at different levels of care, supported by informed, public healthoriented policies and strategies (Shidhaye et al., 2015). In fact, the United Nations has indicated that responding to this need is a high priority for governments and for the international community. The 2030 Agenda for Sustainable Development (United Nations, 2015) includes as a specific target to 'strengthen the prevention and treatment of substance abuse, including narcotic drugs and harmful use of alcohol' (Target 3.5). This is one of only 13 development targets related to all of the health.

\section{Importance of World Health Organization (WHO)'s International Classification of Diseases (ICD)}

The WHO is currently developing the Eleventh Revision of the International Classification of Diseases and Related Health Problems (ICD-11), to be published in 2018. This represents the first major revision of the classification since the World Health Assembly approved the ICD-10 more than 25 years ago (World Health Organization, 1992). The development of the ICD-11 chapter on Mental and Behavioural, including Disorders Due to Substance Use, is being led by the WHO Department of Mental Health and Substance Abuse. The Department has indicated that core priorities in the development of ICD-11 Mental and Behavioural Disorders include providing WHO member states with a better tool for reducing the disease burden of these disorders and providing health professionals with a better tool for identifying people in need of treatment at the point at which they are most likely to come into contact with opportunities for care and for determining which treatments are most likely to be beneficial (International Advisory Group for the Revision of ICD-10 Mental and Behavioural Disorders, 2011). Key principles for the conceptualisation and testing of ICD-11 diagnostic guidelines are clinical utility and global applicability (Keeley et al. 2016).
The ICD-11 provides a major opportunity to contribute to increasing coverage and improving outcomes of prevention and treatment interventions for substance use disorders. In addition to providing the global basis for the collection and reporting of health statistics, the ICD is increasingly used by governments around the world as a key part of the framework for defining their obligations to provide free or subsidised health services to their citizens (International Advisory Group for the Revision of ICD-10 Mental and Behavioural Disorders, 2011). At the same time, national efforts to establish parity between services for mental and substance use disorders and services for other areas of health have been increasingly successful. This means that changes to the ICD have major implications for the conceptualisation, structure and availability of services for substance use disorders across the globe.

\section{A public health approach to the classification of disorders due to substance use}

The proposed ICD-11 approach to the classification of disorders due to substance use is based on the public health approach as a conceptual framework. The public health approach seeks to prevent disease, prolong life and promote health through the organised efforts and informed choices of society, organisations, public and private communities and individuals (Winslow, 1920). As applied to substance use, there has been a substantial shift towards this approach in recent years, particularly evident in international drug policies that adopt the well-being of people and communities rather than drug seizures and arrests as the main indicators of success (United Nations Office on Drugs and Crime, 2016). The U.S. Surgeon General has indicated that until recently there has been limited awareness of the nature of substance use disorders as diseases or health conditions and that drug use has rather been regarded as a social problem or a character defect (U.S. Department of Health and Human Services, 2016). Drug use is still punishable by imprisonment in some countries, and compulsory drug detention centres are still major providers of nonvoluntary 'treatment' in many parts of the world, in spite of evidence of their ineffectiveness (Wegman et al. 2017). However, voluntary treatment is increasingly seen as the best alternative (United Nations Office on Drugs and Crime, 2009).

This shift has led to a growing demand for effective and public health-oriented prevention and treatment interventions and increasing acknowledgement of the role of the health sector and the public health community (Babor et al. 2010). At the same time, 
diversification of psychoactive substances and changes in their routes of administration and the contexts of their use present new challenges for governments and for health systems (United Nations Office on Drugs and Crime, 2017). For example, this is evidenced by the rapid development and diffusion of new, synthetic psychoactive substances, the epidemic of abuse and overdose due to prescription opioids in some parts of the world, and the use of vaporising ('vaping') devices in administering dependenceproducing psychoactive substances (Dawkins et al., 2013; Lee et al., 2016).

The public health approach recognises substance use and substance use disorders as a spectrum of behaviours and health conditions that may require different approaches, services and systems in order to achieve public health objectives. It recognises that not all those who use psychoactive substances have negative health outcomes and that the expression of health conditions due to substance use is derived from the interaction between psychoactive substances and the patterns of their use, the inherited or acquired characteristics of the individuals using them, and the environment. For example, Koob \& Volkow (2016) have described three phases in the development of dependence in a 'cycle that goes from repetition (pursuit of pleasure), to maladaptation (negative emotions and withdrawal), to an increasingly severe dependence (loss of control), which denote repeated failures in self-regulation.' That is, dependence occurs at the end of a long sequence of behavioural and chemical changes, and characterises only a small portion of the population using psychoactive substances at any given time (Lopez-Quintero et al. 2011). From a public health perspective, it is critical that the classification of substance use disorders cover different stages and patterns of substance use through a set of diagnostic categories that are organised as much as possible on a continuum reflecting the stages and severity of substance use disorders. Classification should facilitate recognition of health conditions relevant for prevention, treatment and rehabilitation and help to indicate based on individual needs the type of prevention or treatment intervention that should be applied.

\section{Current status of substance use services}

Currently, there is enormous variation across countries in the structure of services and systems for substance use disorders and the degree to which these services are supported in comparison with other chronic health conditions (World Health Organization, 2014a, b, c). Overall, treatment systems are still very much oriented to advanced cases of substance dependence or management of substance-induced mental disorders and withdrawal syndromes, with substantial delays between the onset of early phase substance use disorders and initial treatment episodes (Wang, AguilarGaxiola et al., 2007; Wang, Angermeyer et al., 2007). Although there have been notable advances in the development of treatment systems for substance use disorders, specialised services are largely absent in many low and middle-income countries or available only in capitals and big cities. The mutual aid movement is still a major source of help, and in many places is the sole form of intervention available. Where available, brief interventions for harmful use have shown positive results (Strang et al. 2012). However, considerable obstacles remain to scale up implementation of screening and early interventions for hazardous patterns of substance use (Babor et al. 2010; Chalk et al., 2010).

In general, there has been a shift from an abstinence model of substance abuse treatment to a more liberal approach aimed at reducing consumption, particularly in the case of alcohol, and developing increased control over consumption and intoxication, partly with the goal of increasing acceptance and coverage of early treatment (van Amsterdam \& van den Brink, 2013). These harm reduction approaches aim to reduce the negative consequences of substance use rather than to eliminate use. Treatment success is conceived of as a reduction in the level of substance-related problems and use episodes, as measured by days of use and severity of symptoms. There is also an increasing emphasis on preventive interventions in diverse health care settings, and screening and brief interventions for hazardous and harmful drinking are now regarded as one of the few costeffective methods of reducing the harmful use of alcohol worldwide (Anderson et al., 2009; Organisation for Economic Co-operation and Development, 2015).

A full spectrum of services that to reduce the health and social burden caused by psychoactive substance use will only be possible with the engagement of health professionals in a broad range of health and social services settings. The traditional separation of services for substance abuse and other health conditions is not been helpful in this regard (U.S. Department of Health and Human Services, 2016). While individuals with substance dependence may require structured and complex biopsychosocial interventions, other patterns of substance use can be assessed and treated in a broad range of nonspecialised health care settings. The classification of disorders due to substance use in the ICD-11 should therefore be suitable for use by a broad range of health professionals to cover the whole spectrum of problems related to psychoactive substance use in order to achieve an impact on population health. 
Innovations in the ICD-11 classification of disorders due to substance use

Based on the current context and global priorities reviewed above, major innovations have been proposed for the classification of disorders due to substance use in the ICD-11. The proposed structure and definitions for ICD-11 diagnostic categories are available on WHO's ICD-11 beta platform (https://icd.who.int/ dev11/l-m/en). Complete diagnostic guidelines for Mental and Behavioural Disorders will be published later by the WHO Department of Mental Health and Substance Abuse (see http://gcp.network). Changes in the classification of Disorders Due to Substance Use were originally proposed the Working Group on the Classification of Substance-Related and Addictive Disorders, a multidisciplinary group of experts from all regions of the world appointed by the WHO Department of Mental Health and Substance Abuse.

Major changes proposed include the following: (1) an updated and expanded range of substance classes; (2) greater specification of different harmful patterns of substance use, which may be continuous or episodic and recurrent; (3) a new category to denote single episodes of harmful use; (4) a category describing hazardous use of substances, which is not considered to be a disorder, but is proposed for inclusion in the ICD-11 chapter on 'Factors influencing health status and encounters with health services'; and (5) simplification of diagnostic guidelines for substance dependence. These changes and the opportunities they present for prevention, treatment and improved monitoring are described briefly in the following paragraphs.

\section{Updated and expanded classification of substance classes}

The updated and expanded range of substance classes proposed for classification in the ICD-11 reflects changes in substances increasingly associated with public health consequences of substance use in different parts of the world. This will support more accurate tracking in health systems and therefore in aggregated health data as a basis for the formulation of appropriate clinical, public health and social policy responses at national and global levels. Specifically, anxiolytic substances have been explicitly mentioned as a part of the class of sedative and hypnotic substances in line with current WHO nomenclature and the dependence-producing properties of these substances. Caffeine is separated from other stimulants because of the increasing public health importance of certain forms of caffeine use, such as the use of energy drinks containing extremely high doses of caffeine, which are generally unregulated at present, or mixtures of methamphetamine with caffeine. Tobacco in ICD-10 is replaced by nicotine in ICD-11, reflecting the increasing use of alternate forms of nicotine, such as through vaporisers. MDMA or 'ecstasy' and related substances and dissociative drugs such as ketamine and PCP are proposed as separate classes of psychoactive substances in the ICD-11. In view of the increasing public health importance of so-called new psychoactive substances (European Monitoring Centre for Drugs and Drug Addiction and Eurojust, 2016), synthetic cannabinoids and synthetic cathinones have been proposed as new classes of psychoactive substances in the ICD-11. In this way, the use of synthetic cannabinoids can be tracked separately from cannabis and cannabis resin.

\section{Harmful pattern of use}

As proposed for the ICD-11, 'harmful use' in the ICD-10 is replaced by 'harmful pattern of use', which can be further specified as continuous or episodic. Importantly, harm to the health of others has been included in the definition of harmful use. This reflects the increasing role of this type of harm in shaping policy and program responses, which is particularly prominent in relation to tobacco smoking. Harm to the health of others may be intentional, such as in case of homicides or interpersonal violence during intoxication with alcohol or drugs, or result from the substance user's difficulty in performing social, professional or family roles, as in the case of negligence towards minors. Damage to the health of others is also being evaluated for possible use in improving the measurement of disease burden caused by substance use and the overall costs of substance use for societies. As proposed for ICD-11, a harmful pattern of use is defined as one that has caused clinically significant harm to a person's physical or mental health or in which substance-induced behaviour has caused clinically significant harm to the health of other people. Again, harm may be caused by the intoxicating effects of a substance, the direct or secondary toxic effects on body organs and systems, or a harmful route of administration.

\section{Single episode of harmful use}

A new diagnostic category 'single episode of harmful use' has been proposed for the ICD-11, with the objective of facilitating recognition of substance use episodes that result in harm to health in the absence of the diagnostic features of substance dependence or a harmful pattern of substance use. This category is specifically intended for use in the context of a wide range of health services, particularly in primary care and emergency settings, more than in specialised treatment centers for mental or substance use disorders. The proposed definition is equivalent to the definition for a harmful pattern of use except that the harm has been caused by a single episode of use. 
The inclusion and expansion of the concept of harmful use as proposed for ICD-11 are extremely important because it provides opportunities for prevention as well as early recognition of relevant behaviours related to substance use. These forms of problems can be preclinical in comparison to how substance use disorders are encountered in specialty settings. The identification of harmful use, either as a pattern or as a single episode, will also help to identify individuals who are likely to respond to brief psychological interventions delivered by non-specialist health care providers. Through the aggregation of health encounter data, these categories can also support better monitoring of the impact of substance use on population health.

\section{Hazardous use of substances}

Hazardous use of substances is a concept that has long been integrated into WHO's approach to problems related to substance use. This category has been proposed for inclusion in the ICD-11 not because it represents a mental or behavioural disorder but rather because it is a behaviour that requires health actions. Accordingly, it has been proposed for inclusion with other phenomena relevant to health status and health interventions that do not represent disorders or diseases in the ICD-11 chapter on 'Factors influencing health status and encounters with health services'. As proposed for ICD-11, hazardous use is defined as 'a pattern of psychoactive substance use that appreciably increases the risk of harmful physical or mental health consequences to the user or to others to an extent that warrants attention and advice from health professionals. The increased risk may be from the frequency of substance use, from the amount used on a given occasion, from risky behaviours associated with substance use or the context of use, from a harmful route of administration, or from a combination of these. The risk may be related to short-term effects of the substance or to longer-term cumulative effects on physical or mental health or functioning. Hazardous substance use has not yet reached the level of having caused harm to the physical or mental health of the user or others around the user.' Hazardous use is an appropriate target for the simplest, briefest interventions focused on prevention of substance use and its negative health consequences and the prevention of progression to harmful substance use and dependence.

\section{Simplification of diagnostic guidelines for substance dependence}

As proposed for the ICD-11, the diagnostic requirements for substance dependence have been simplified in comparison to ICD-10. The key feature of substance dependence is a strong internal drive to use the substance in question, manifested by impaired ability to control use and increasing priority given to substance use over other activities. Individuals with substance dependence often exhibit physiological features such as the development of tolerance and withdrawal symptoms. In order to assign the diagnosis, the constellation of behaviours suggesting dependence should be evident over a period of at least 12 months if use is episodic, or over a period of at least one month if use is continuous (daily or almost daily).

\section{Conclusion}

The proposed classification of substance use disorders and problems in ICD-11 includes a range of diagnostic categories that cover a broad spectrum of health conditions reflecting different levels and patterns of substance use. A major goal of this system is to facilitate early recognition of the negative impact of substance use on health and the provision of targeted prevention and treatment interventions at various levels of care corresponding to the needs and levels of harm caused by substance use. The proposed changes in ICD-11 are also intended to increase the capacity of WHO member states to monitor the health consequences of substance use at a population level.

We believe that the ICD-11 can be a useful tool in reducing the treatment gap between those who may benefit from prevention and treatment interventions and those who actually receive them and, over time, to improve treatment coverage for substance use disorders. Implementation of the new classification can also improve the capacity of health systems to generate useful data based on health encounters that can be aggregated at the system, local, national and global level to support effective planning and the development of treatment systems for substance use disorders. The concepts and definitions for problems related to substance use proposed for the ICD-11 will also help to influence relevant health policies through the promotion of a public health approach to substance use disorders and the engagement of a broad spectrum of health professionals at different levels of health systems in their identification and management.

\section{Acknowledgements}

The ICD-11 Working Group on the Classification of Substance-Related and Addictive Disorders was responsible for the initial development of the proposals discussed in this article. The Working Group included Rajat Ray (Chair), Sawitri Assanangkornchai, Thomas 
Babor, Miguel Casas, Karl Mann, Neo Morojele, Marina Piazza, Afarin Rahimi, John Saunders and Wei Hao.

\section{Financial support}

The participation of M.E. Medina-Mora and G.M. Reed on this article was funded by the National Institute of Psychiatry Ramón de la Fuente and by the National Council on Science and Technology (CONACyT), Mexico.

\section{Conflict of interest}

None.

\section{Author Note}

V. Poznyak and G.M. Reed are members of the WHO Secretariat, Department of Mental Health and Substance Abuse. M.E. Medina-Mora is a member of the WHO International Advisory Group for the Revision of ICD-10 Mental and Behavioural Disorders and the Field Studies Coordinating Group for ICD-11 Mental and Behavioural Disorders. The views expressed in this article are those of the authors and, unless specifically noted, do not represent the official policies or positions of the World Health Organization.

\section{References}

Anderson P, Chisholm D, Fuhr D (2009). Effectiveness and cost-effectiveness of policies and programmes to reduce the harm caused by alcohol. Lancet 373, 2234-2246. doi: 10.1016/S0140-6736(09)60744-3.

Babor T, Caulkins JP, Edwards G, Fischer B, Foxcroft DR, Humphreys K, Obot IS, Rehm J, Reuter P, Room R, Rossow I, Strang J (2010). Drug Policy and the Public Good. Oxford University Press: Oxford, UK.

Chalk M, Dilonardo J, Rinaldo SG, Oehlmann P (2010). Integrating Appropriate Services for Substance use Conditions in Health Care Settings: An Issue Brief on Lessons Learned and Challenges Ahead. Treatment Research Institute: Philadelphia, PA.

Chisholm D, Saxena S. (2012). Cost effectiveness of strategies to combat neuropsychiatric conditions in sub-Saharan Africa and South East Asia: Mathematical modelling study. BMJ 344, e609. doi: 10.1136/bmj.e609.

Dawkins L, Turner J, Roberts A, Soar K (2013). 'Vaping' profiles and preferences: an online survey of electronic cigarette users. Addiction 108, 1115-1125. doi: 10.1111/ add. 12150
European Monitoring Centre for Drugs and Drug Addiction and Eurojust (2016). New Psychoactive Substances in Europe: Legislation and Prosecution - Current Challenges and Solution. European Monitoring Centre for Drugs and Drug Addiction and Eurojust: Lisbon, Portugal. Retrieved from http://www.emcdda.europa.eu/publications/jointpublications/eurojust/nps-legislation-and-prosecution_en.

GBD 2015 Risk Factors Collaborators (2016). Global, regional, and national comparative risk assessment of 79 behavioural, environmental and occupational, and metabolic risks or clusters of risks, 1990-2015: a systematic analysis for the Global Burden of Disease Study 2015. Lancet 388, 1659-1724. doi: 10.1016/S0140-6736(16)31679-8. International Advisory Group for the Revision of ICD-10 Mental and Behavioural Disorders (2011). A conceptual framework for the revision of the ICD-10 classification of mental and behavioural disorders. World Psychiatry 10, 8692. doi: 10.1002/j.2051-5545.2011.tb00022.x.

Keeley JW, Reed GM, Roberts MC, Evans SC, Medina-Mora ME, Robles R, Rebello T, Sharan P, Gureje O, First MB, Andrews HF, Ayuso-Mateos JL, Gaebel W, Zielasek J, Saxena S (2016). Developing a science of clinical utility in diagnostic classification systems: field study strategies for ICD-11 Mental and Behavioural Disorders. American Psychologist 71, 3-16. doi: 10.1037/a0039972.

Koob GF, Volkow N (2016). Neurobiology of addiction: a neurocircuitry analysis. Lancet Psychiatry 3, 760-773. doi: 10.1016/S2215-0366(16)00104-8.

Lee D, Crosier B, Borodovsky J, Sargent J, Budney A (2016). Online survey characterizing vaporizer use among cannabis users. Drug and Alcohol Dependence 159, 227-233. doi: 10.1016/j.drugalcdep.2015.12.020.

Lopez-Quintero C, Pérez de los Cobos J, Hasin DS, Okuda M, Wang S, Grant BF, Bianco C (2011) Probability and predictors of transition from first use to dependence on nicotine, alcohol, cannabis, and cocaine: Results of the National Epidemiologic Survey on Alcohol and Related Conditions (NESARC). Drug and Alcohol Dependence 115, 120-130. doi: 10.1016/j.drugalcdep.2010.11.004.

McLellan AT, Lewis DC, O'Brien CP, Kleber HD (2000). Drug dependence, a chronic medical illness: implications for treatment, insurance, and outcomes evaluation. JAMA 284, 1689-1695.

Organisation for Economic Co-operation and Development (2015). Tackling Harmful Alcohol Use: Economics and Public Health Policy. OECD Publishing: Paris. doi: 10.1787/ 9789264181069-en.

Shidhaye R, Lund C, Chisholm D (2015). Closing the treatment gap for mental, neurological and substance use disorders by strengthening existing health care platforms: strategies for delivery and integration of evidence-based interventions. International Journal of Mental Health Systems 9, 40. doi: 10.1186/s13033-015-0031-9.

Strang J, Babor T, Caulkins J, Fischer B, Foxcroft D, Humphreys K (2012). Drug policy and the public good: evidence for effective interventions. Lancet 379, 71-83. doi: 10.1016/S0140-6736(11)61674-7.

United Nations (2015). Transforming our World: The 2030 Agenda for Sustainable Development. United Nations: 
New York. Retrieved from https://sustainabledevelopment. un.org/post2015/transformingourworld.

United Nations Office on Drugs and Crime (2009). From Coercion to Cohesion: Treating Drug Dependence Through Health Care, not Punishment. United Nations Office on Drugs and Crime: Vienna, Austria. Retrieved from https:// www.unodc.org/docs/treatment/Coercion_Ebook.pdf.

United Nations Office on Drugs and Crime (2016). Outcome Document of the 2016 United Nations General Assembly Special Session on the World Drug Problem: Our Joint Commitment to Effectively Addressing and Countering the World Drug Problem. United Nations Office on Drugs and Crime: Vienna, Austria. Retrieved from https://www.unodc.org/ documents/postungass2016/outcome/V1603301-E.pdf.

United Nations Office on Drugs and Crime (2017). World Drug Report 2017. United Nations Office on Drugs and Crime: Vienna, Austria. Retrieved from https://www. unodc.org/wdr2017/index.html.

U.S. Department of Health and Human Services (HHS), Office of the Surgeon General (2016). Facing Addiction in America: The Surgeon General's Report on Alcohol, Drugs, and Health. U.S. Department of Health and Human Services (HHS), Office of the Surgeon General: Washington, DC. Retrieved from https:// addiction.surgeongeneral.gov/surgeon-generals-report.pdf.

van Amsterdam J, van den Brink W (2013). Reduced risk drinking as a viable treatment goal in problematic alcohol use and alcohol dependence. Journal of Psychopharmacology 27, 987-997. doi: 10.1177/0269881113495320.

Wang PS, Aguilar-Gaxiola S, Alonso J, Angermeyer MC, Borges G, Bromet EJ, Bruffaerts R, de Girolamo G, de Graaf R, Gureje O, Haro JM, Karam EG, Kessler RC, Kovess V, Lane MC, Lee S, Levinson D, Ono $Y$, Petukhova M, Posada-Villa J, Seedat S, Wells JE (2007). Worldwide use of mental health services for anxiety, mood, and substance disorders: results from 17 countries in the WHO World Mental Health (WMH) Surveys. Lancet 370, 841-850. doi: 10.1016/S0140-6736(07)61414-7.

Wang PS, Angermeyer M, Borges G, Bruffaerts R, Chiu WT, de Girolamo G, Fayyad J, Gureje O, Haro JM, Huang Y, Kessler RC, Kovess V, Levinson D, Nakane Y, Oakley
Brown MA, Ormel JH, Posada-Villa J, Aguilar-Gaxiola S, Alonso J, Lee S, Heeringa S, Pennell BE, Chatterji S, Üstün TB (2007). Delay and failure in treatment seeking after first onset of mental disorders in the World Health Organization's World Mental Health Survey Initiative. World Psychiatry 6, 177-185.

Wegman MP, Altice FL, Kaur S, Rajandaran V, Osornprasop S, Wilson D, Wilson DP, Kamarulzaman A (2017) Relapse to opioid use in opioid-dependent individuals released from compulsory drug detention centres compared with those from voluntary methadone treatment centres in Malaysia: a two-arm, prospective observational study. Lancet Global Health 5, e198-e207. doi: 10.1016/S2214-109X(16)30303-5.

Winslow CE (1920). The untilled fields of public health. Science 51, 23-33. doi: 10.1126/science.51.1306.23.

World Health Organization (1992). The ICD-10 Classification of Mental and Behavioural Disorders: Clinical Descriptions and Diagnostic Guidelines. World Health Organization: Geneva, Switzerland.

World Health Organization (2004). Neuroscience of Psychoactive Substance use and Dependence. Geneva, Switzerland.

World Health Organization (2014a). Global Status Report on Alcohol and Health. World Health Organization: Geneva, Switzerland. Retrieved from http://www.who.int/ substance_abuse/publications/global_alcohol_report/en/.

World Health Organization (2014b). Integrating the Response to Mental Disorders and Other Chronic Diseases in Health Care Systems. World Health Organization: Geneva, Switzerland. Retrieved from http://apps.who.int/iris/bitstream/10665/ 112830/1/9789241506793_eng.pdf.

World Health Organization (2014c). Mental Health Atlas 2014. World Health Organization: Geneva, Switzerland. Retrieved from http://www.who.int/mental_health/ evidence/atlas/mental_health_atlas_2014/en/.

World Health Organization (2015). WHO Report on the Global Tobacco Epidemic: Raising Taxes on Tobacco. Geneva, Switzerland. Retrieved from http://www.who.int/tobacco/ global_report/2015/en/. 